\title{
Myocardial perfusion scintigraphy dosimetry: optimal use of SPECT and SPECT/CT technologies in stress-first imaging protocol
}

\author{
M. Lecchi ${ }^{1,2} \cdot$ S. Malaspina ${ }^{3} \cdot$ C. Scabbio ${ }^{1} \cdot$ V. Gaudieri ${ }^{4} \cdot$ A. Del Sole $^{2,3}$ D
}

Received: 27 September 2016/ Accepted: 20 October 2016/Published online: 31 October 2016

(C) The Author(s) 2016. This article is published with open access at Springerlink.com

\begin{abstract}
Purpose Over the past decade, nuclear medicine experts have been seeking to minimize patient exposure to radiation in myocardial perfusion scintigraphy (MPS). This review describes the latest technological innovations in MPS, particularly with regard to dose reduction.

Methods We searched in PubMed for original clinical papers in English, published after 2008, using the following research criteria: (dose) and ((reduction) or (reducing)) and ((myocardial) or (cardiac) or (heart)) and ((nuclear medicine) or (nuclear imaging) or (radionuclide) or (scintigraphy) or (SPET) or (SPECT)). Thereafter, recent reviews on the topic were considered and other relevant clinical papers were added to the results.

Results Of 202 non-duplicate articles, 17 were included. To these, another eight papers cited in recent reviews were added. By optimizing the features of software, i.e., through algorithms for iterative reconstruction with resolution recovery (IRRs), and hardware, i.e., scanners and collimators, and by preferring, unless otherwise indicated, the use of stress-first imaging protocols, it has become possible to reduce the effective dose by at least $50 \%$ in stress/rest protocols, and by up to $89 \%$ in patients undergoing a
\end{abstract}

A. Del Sole

angelo.delsole@unimi.it

1 Health Physics, San Paolo Hospital, Via Antonio di Rudini, 20142 Milan, Italy

2 Department of Health Sciences, University of Milan, Milan, Italy

3 Nuclear Medicine Unit, Department of Diagnostic Services, ASST Santi Paolo e Carlo, Milan, Italy

4 Department of Advanced Biomedical Sciences, University Federico II, Naples, Italy diagnostic stress-only study with new technology. With today's SPECT/CT systems, the use of a stress-first protocol can conveniently be performed, resulting in an overall dose reduction of about $35 \%$ if two-thirds of stressfirst examinations were considered definitively normal. Conclusion Using innovative gamma cameras, collimators and software, as well as, unless otherwise indicated, stressfirst imaging protocols, it has become possible to reduce significantly the effective dose in a high percentage of patients, even when X-ray CT scanning is performed for attenuation correction.

Keywords Myocardial perfusion imaging - SPECT - CT · Dose reduction - Patient dose - Radiation exposure

\section{Introduction}

Radiological and nuclear medicine procedures are the leading cause of population radiation exposure in Western countries, and there is concern over their potential longterm effects on patient health [1]. Even though, based on the linear no-threshold model, radiation-induced cancer at low doses is probabilistic, it has been demonstrated that this risk increases after exposure to a cumulative dose of radiation greater than $100 \mathrm{mSv}$ [2], a level that can easily be reached through repeated investigations with ionizing radiation.

Among the various procedures involving the use of ionizing radiation, myocardial perfusion scintigraphy (MPS) alone is responsible for over $22 \%$ of the total effective dose from all medical imaging procedures in the United States [3]. Therefore, over the past decade, minimization of patient exposure to radiation in nuclear cardiology has become a priority not only for nuclear medicine 
physicians and health physicists, but also for technologists who perform these scans on a daily basis $[4,5]$. This objective can be pursued by adhering to the two main principles of radiation protection: justification of the procedure and optimization of the patient dose in relation to the available technology.

It is to be noted that the prevalence of non-pathological MPS findings may be as high as $35 \%$ in patients with known coronary artery disease (CAD) and as high as $81 \%$ in those without known CAD [6]. Therefore, conservative strategies promoting radiation protection, such as the use of "stress-first" studies, should, providing there is adequate justification, be preferred for the evaluation of patients, since subsequent rest studies may be avoided in those with a negative stress MPS (stress-only protocol).

The replacement (with some specific exceptions) of ${ }^{201} \mathrm{Tl}$-chloride with two ${ }^{99 \mathrm{~m}} \mathrm{Tc}$-labeled radiopharmaceuticals (physical half-time $=6 \mathrm{~h}$ ), namely ${ }^{99 \mathrm{~m}} \mathrm{Tc}$-sestamibi and ${ }^{99 \mathrm{~m}} \mathrm{Tc}$-tetrofosmi, has made a major contribution to dose optimization in MPS. More recently, the possibility of carrying out the image acquisition with cardiac dedicated systems rather than with general-purpose (GP) gamma cameras has emerged, and this represents another step forward [7].

Depending on the clinical question and the camera used, MPS studies may require either one acquisition under stress, or two acquisitions (one under stress and one under rest). Accordingly, the imaging protocol may be a one-day stress-only study (one acquisition) or either a two-day imaging protocol or a one-day stress-first protocol (each involving two acquisitions) [8]. Moreover, if a patient has a large body size, this can result in a higher proportion of emitted photons being attenuated within the patient. Therefore, clinical MPS protocols should take into account the patient's weight or body mass index (BMI) [9].

The optimal amount of activity to be administered to the single patient is determined taking all of the above variables into account. On this basis, the recommended activities, per single scan, according to the American Society of Nuclear Cardiology (ASNC), may range from $148 \mathrm{MBq}$ (stress-only protocol, new technology, BMI $=25 \mathrm{~kg} / \mathrm{m}^{2}$ ) to $1332 \mathrm{MBq}$ (second injection in a one-day stress/rest protocol, GP gamma camera, BMI $>35 \mathrm{~kg} / \mathrm{m}^{2}$ ), resulting in effective doses of between $1.0 \mathrm{mSv}$ and $10.5 \mathrm{mSv}$ [7]. When rest and stress studies are both performed on the same day, the MPS dose may be as low as $4.5 \mathrm{mSv}$ in subjects with $\mathrm{BMI}=25 \mathrm{~kg} / \mathrm{m}^{2}$, providing the recommended procedure is followed and new technologies are employed; conversely, the patient dose can reach $13.5 \mathrm{mSv}$ when using a GP gamma camera in subjects with a BMI $>35 \mathrm{~kg} / \mathrm{m}^{2}$.

Therefore, in this setting, the use of innovative gamma cameras, collimators and software is crucial. By optimizing the features of the software, i.e., through algorithms for iterative reconstruction with resolution recovery (IRRs), and hardware, i.e., the scanners and collimators, and by preferring, unless otherwise indicated, the use of stress-first imaging protocols, it has become possible to reduce the effective dose by at least $50 \%$ in stress/rest protocols, and by up to $89 \%$ in patients undergoing a diagnostic stressonly study with new technology.

The development of high-efficiency cardiac dedicated scanners equipped with cadmium-zinc-telluride (CZT) detectors has been paralleled by the development of single photon emission computed tomography (SPECT) systems combined with X-ray computed tomography (CT) scanners, mostly employed for attenuation correction (AC) of the emission data. Thus, although the advanced technologies may allow reductions in patient doses, the new combined scanners, due to the CT component, might actually lead to an increase in radiation exposure [10]. The optimized dose from a single CT scan for $\mathrm{AC}$ is in the order of $0.3-1.3 \mathrm{mSv}$ [11] and, therefore, it is not negligible, when compared with the dose from the injected radiopharmaceutical, especially for low-dose protocols. Whether or not AC is actually needed is a long-debated and still unresolved question [12], which applies not only to MPS but also other cardiac SPECT examinations, such as evaluation of the sympathetic innervation [13].

The impact of stress-first/stress-only protocols and new technologies, with and without CT, on the patient dose in MPS studies are summarized in Table 1 for normal-weight patients and in Table 2 for obese patients. These doses are based on the very recent ASNC imaging guidelines [7] and on epidemiological data available from a large cohort study [6].

The features of the new technologies in relation to dose optimization are described in the following paragraphs.

\section{Dose reduction in MPS: IRR algorithms}

MPS is commonly performed using GP dual-head gamma cameras based on $\mathrm{NaI}(\mathrm{Tl})$ scintillation detectors with photomultiplier tube array and high-resolution parallelhole collimators. However, limitations of these SPECT systems-namely their low count sensitivity and poor spatial resolution-mean that it is necessary to administer activities in the range of $296-444 \mathrm{MBq}$ for the first radiopharmaceutical injection, and three times as much for the second injection on the same day, and in most cases to perform both rest and stress studies, due to the low image quality and presence of attenuation artifacts when no form of AC is applied (e.g., Gd-153 line source, CT or supine and prone imaging) [14]. But this procedure may entail injected total activities (summing both rest and stress injected activities) of 1.184-1.776 MBq and doses of up to $13.5 \mathrm{mSv}$ per patient, with acquisitions lasting 
Table 1 Mean effective dose among different imaging protocols and SPECT technologies, with and without CT, in normal-weight patients undergoing MPS studies with a normal rate of 66\%. Data from [6] and [10]

\begin{tabular}{|c|c|c|c|c|}
\hline Patient size & Protocol & Conventional gamma camera & Newer technology & New technology $+\mathrm{CT}^{* * *}$ \\
\hline \multirow[t]{6}{*}{$\mathrm{BMI}=25 \mathrm{~kg} / \mathrm{m}^{2}$} & For all patients & \multirow[t]{2}{*}{$9.0 \mathrm{mSv}(\mathrm{A})$} & $4.5 \mathrm{mSv}(\mathrm{B})$ & $5.5 \mathrm{mSv}$ \\
\hline & 1-day stress/rest & & $-50 \%$ vs $\mathrm{A}$ & $\begin{array}{l}-39 \% \text { vs } \mathrm{A} \\
+22 \% \text { vs } \mathrm{B}\end{array}$ \\
\hline & $1 / 3$ patients & \multirow[t]{4}{*}{ Not used } & $2.2 * * \mathrm{mSv}$ & $3.2 \mathrm{mSv}$ \\
\hline & \multirow{2}{*}{$\begin{array}{l}1 \text { day stress/rest } \\
\text { plus }\end{array}$} & & $-76 \%$ vs $\mathrm{A}$ & $-64 \%$ vs $\mathrm{A}$ \\
\hline & & & $-51 \%$ vs B & $-29 \%$ vs B \\
\hline & $\begin{array}{l}2 / 3 \text { patients } \\
\text { stress only* }\end{array}$ & & & \\
\hline
\end{tabular}

\footnotetext{
* Stress dose $=1 \mathrm{mSv}$

** Used with prone-supine or upright-supine imaging

*** CT dose of $1 \mathrm{mSv}$ for stress study (total stress dose $=2 \mathrm{mSv}$ )
}

Table 2 Mean effective dose among different imaging protocols and SPECT technologies, with and without CT, for obese patients undergoing MPS studies with a normal rate of $66 \%$. Data from [6] and [10]

\begin{tabular}{|c|c|c|c|c|}
\hline Patient size & Protocol & Conventional gamma camera & Newer technology & New technology $+\mathrm{CT}^{* * * *}$ \\
\hline \multirow[t]{6}{*}{$\mathrm{BMI}>35 \mathrm{~kg} / \mathrm{m}^{2}$} & For all patients & $13.5 \mathrm{mSv}(\mathrm{A})$ & $6.7 \mathrm{mSv}(\mathrm{B})$ & $7.7 \mathrm{mSv}$ \\
\hline & 1-day stress/rest & & $-50 \%$ vs A & $-43 \%$ vs A \\
\hline & & & & $+15 \%$ vs $\mathrm{B}$ \\
\hline & $1 / 3$ of patients & Not used & $3.2 * * \mathrm{mSv}$ & $4.2 \mathrm{mSv}$ \\
\hline & 1 day stress/rest plus & & $-76 \%$ vs A & $-69 \%$ vs A \\
\hline & $2 / 3$ of patients stress only* & & $-52 \%$ vs B & $-37 \%$ vs $\mathrm{B}$ \\
\hline
\end{tabular}

\footnotetext{
* Stress dose $=1.5 \mathrm{mSv}$

** Used with prone-supine or upright-supine imaging

$* * * \mathrm{CT}$ dose of $1 \mathrm{mSv}$ for stress study (total stress dose $=2.5 \mathrm{mSv}$ )
}

approximately for $15 \mathrm{~min}$, and overall study times of up to $4 \mathrm{~h}$ per patient in the case of one-day stress/rest protocols.

The IRR algorithms include resolution recovery and noise compensation in the iterative reconstruction (IR) process, and they make it possible to reduce the study count statistics, without degradation of the image quality, to $25 \%$ of the reference value of traditional IR algorithms, such as orderedsubset expectation maximization (OSEM) [15]. The use of CT-based AC results in a further reduction of background noise and in an increase of uniformity also in the polar-map representation of the left ventricular signal [16] (Figs. 1,2).

The IRR algorithms were introduced into clinical practice primarily to reduce acquisition times, since fast imaging provides immediate benefits in terms of patient throughput and patient comfort. However, equivalent dose reduction is also possible by performing standard time acquisitions with lower injected activities.

Using the IRR algorithm Astonish ${ }^{\mathrm{TM}}$ (Philips), the possibility of decreasing the dose to $25 \%$ of the reference value was evaluated in the context of a two-day imaging protocol, without $\mathrm{AC}$ ( $3 \mathrm{mSv}$ for a full examination). The authors found that decreasing radiopharmaceutical activity to $25 \%$ of the reference value seemed practicable for normal-weight patients, while an activity reduction limited to $50 \%$ was suggested to be more appropriate for overweight and obese patients [17]. Other studies investigated the possibility of halving the dose to patients using the reduced acquisition time to simulate an equivalent reduction of the injected activity [18-20].

Bateman et al., using the Astonish ${ }^{\mathrm{TM}}$, performed stressonly imaging with Gd-153 for AC and half-counts, and found this method to provide clinical results equivalent to those of conventional stress/rest scanning without AC [21]. Marcassa et al. [22] used wide-beam reconstruction (WBR) to compare patient effective dose in different dose administration protocols. They found that the additional use of an IRR algorithm made it possible to reduce patient effective dose by $40 \%$ (from 9.5 to $5.7 \mathrm{mSv}, p<0.001$ ).

With full-time acquisitions (15 min), a $50 \%$ dose reduction seems to be the limit for IRR algorithms. With today's SPECT/CT systems the use of a stress-first protocol can conveniently be performed. If two-thirds of 

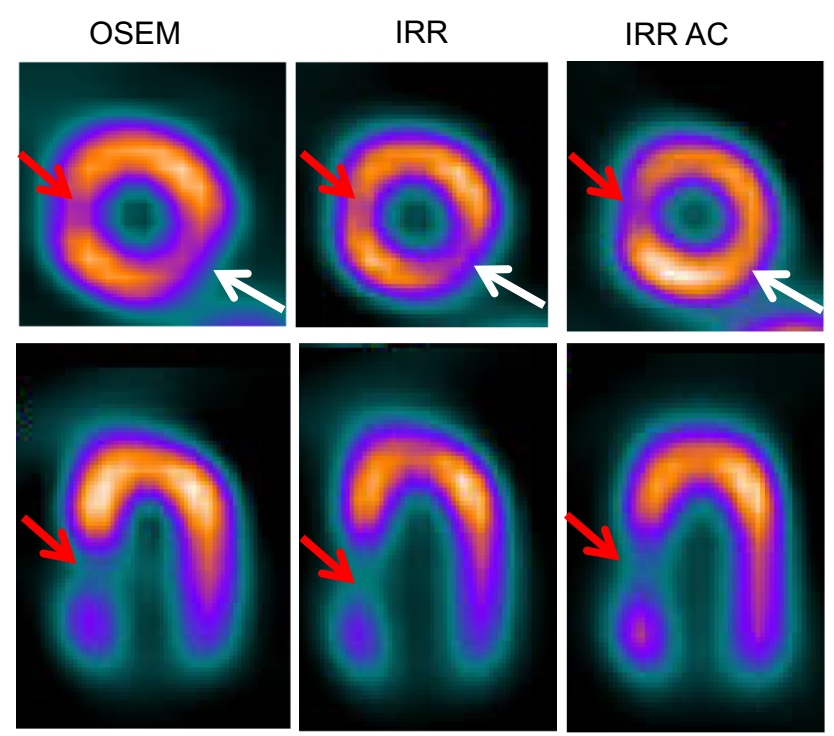

Fig. 1 Comparison between different reconstruction protocols for left ventricular short-axis (top) and horizontal long-axis (bottom) images of an anthropomorphic phantom scan with in vivo reference count statistics (Torso Phantom ${ }^{\mathrm{TM}}$ and Cardiac Insert ${ }^{\mathrm{TM}}$, Data Spectrum Corporation). The white arrows show an attenuation artifact in the uncorrected images. The red arrows show a true perfusion defect in the phantom. OSEM ordered-subset expectation maximization, $I R R$ iterative reconstruction with resolution recovery, $N C$ no correction, $A C$ attenuation correction

patients were considered definitively normal after the stress study, there would be no need to perform the rest study in these patients. In such a situation, patient dose would decrease by $76 \%$, but the CT dose has to be added, resulting in an overall dose reduction of about $35 \%$ (see Tables 1, 2).

\section{MPS dose reduction: multifocal collimators}

An alternative approach, such as the IQ-SPECT solution (Siemens), is to adapt a GP gamma camera to the particular application of cardiac imaging using dedicated multifocal collimators (SMARTZOOM), cardio-centric acquisition, and an IRR algorithm optimized for myocardial studies. Compared with conventional parallel collimation, IQ·SPECT shows similar global image quality, including resolution, but a fourfold higher sensitivity, which allows the use of either a low-dose or a short-time imaging protocol, or a combination of the two [23].

However, attenuation artifacts are more marked than with parallel collimation, as well as being unpredictable and position dependent; for this reason, $\mathrm{AC}$ is mandatory for the IQ.SPECT users [24].

Very recently, Lyon et al., using IQ.SPECT technology, found that one-day rest-stress gated SPECT/CT, quantitative stress MPS imaging is possible with $50 \%$ of the standard injected activity in $50 \%$ of the time with respect to imaging using conventional gamma cameras [25].

To the best of our knowledge, no studies have been performed with IQ.SPECT configuration to evaluate the feasibility of stress-first/stress-only imaging protocol.

\section{MPS dose reduction: CZT detectors}

Technological advances have led to the development of solid-state detectors, in particular CZT tomographs equipped with detector geometry optimized for cardiac imaging, which allow a greater count sensitivity (up to 7 times) with improved spatial resolution (over 2 times) compared with GP
Fig. 2 Left ventricular horizontal long-axis images of an anthropomorphic phantom (Torso Phantom $^{\mathrm{TM}}$ and Cardiac Insert $^{\mathrm{TM}}$, Data Spectrum Corporation) acquired at different potential percentages of dose reduction. Images are reconstructed with iterative reconstruction algorithm with resolution recovery plus scatter and attenuation corrections (top) and with filtered back projection reconstruction (bottom)

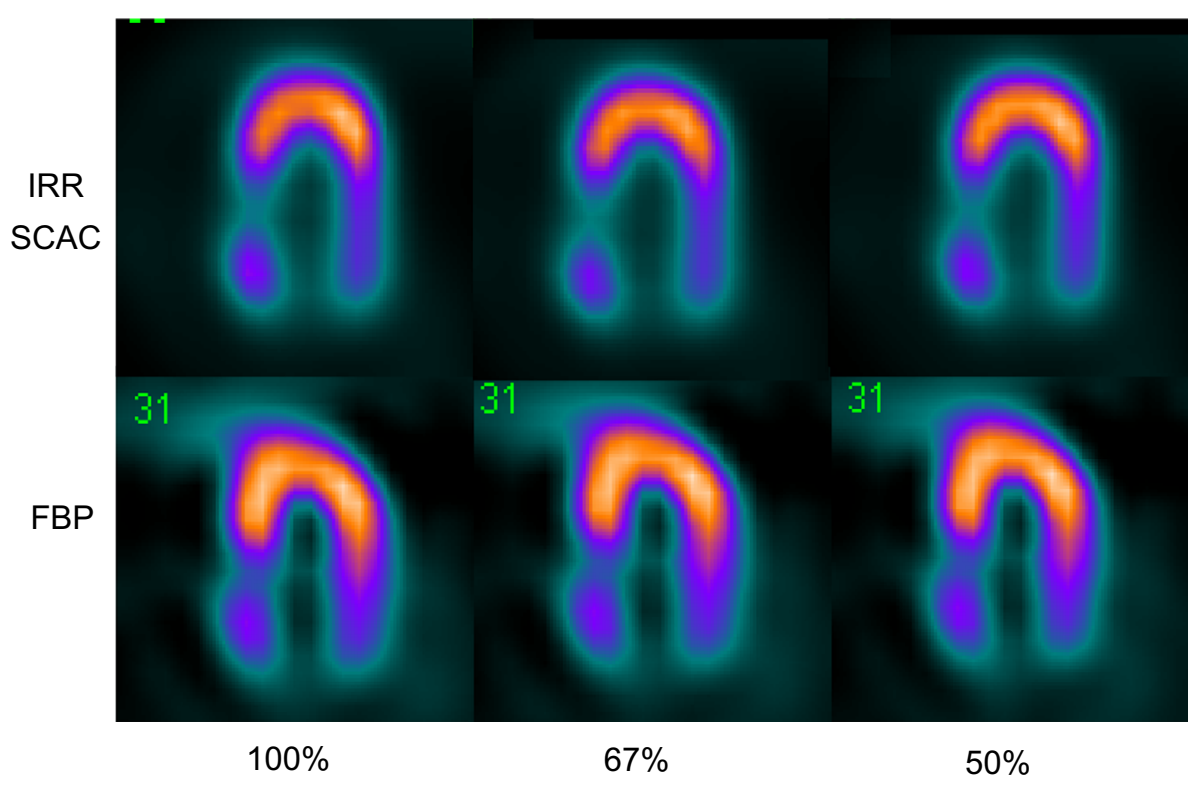

Potential reduction of patient dose 
cameras [26]. Thanks to this sensitivity, CZT cameras provide several advantages and opportunities over GP cameras. First, they make it possible to reduce either the amount of injected activity or the acquisition time, or both, and thus to optimize both. Second, due to the improved image quality of the stress study, i.e., their fewer artifacts, stress studies have a higher prognostic value and allow the rest study to be avoided in the presence of conclusive normal stress scans.

Two systems (Discovery NM 530c, General Electric and D-SPECT, Spectrum Dynamics) having the same configuration as multiple CZT detectors, but coupled with different high-sensitivity collimators (multipinhole vs highsensitivity parallel-hole collimators, respectively) and IRR algorithms, have been introduced on the market [27, 28]. A comparative study, performed with an anthropomorphic phantom mimicking the thorax of a normal adult, has shown relatively similar physical performance of the two CZT cameras, even though the corresponding images of the left ventricle insert were significantly different, revealing a smaller wall thickness with the D-SPECT than with the Discovery NM 530c (13.6 \pm 0.7 vs $19.6 \pm 1.3 \mathrm{~mm}$, respectively) [29]. It is unlikely, however, that such differences have a significant impact on diagnostic accuracy.
The study by Duvall et al. was among the first to demonstrate that a low-dose one-day ${ }^{99 \mathrm{~m}}$ Tc-sestamibi protocol (185 MBq for rest and $555 \mathrm{MBq}$ for stress) with 5-min acquisition time is feasible with the Discovery NM530c, without compromising image quality and diagnostic accuracy. They showed that effective doses to patients could be reduced by $50 \%$ compared to those associated with a conventional gamma camera [30].

Other studies have confirmed the performance of CZT systems in halving the patient dose and, at the same time, in reducing the acquisition time to less than $10 \mathrm{~min}$ (lowdose ultrafast protocol) [31-33].

The possibility of using different patient positions, such as prone or upright, alone or in addition to the supine position, to reduce attenuation artifacts, due to the individual patient's body conformation and weight, was successfully investigated in stress-first procedures and one-day protocols with CZT systems (Figs. 3, 4) [34, 35].

Einstein et al. demonstrated that a stress-only imaging protocol using an advanced CZT system and a fixed activity could be performed in more than two-thirds of patients with an effective dose averaging $1 \mathrm{mSv}$, in a study time that, on average, amounted to less than $2 \mathrm{~h}$ to
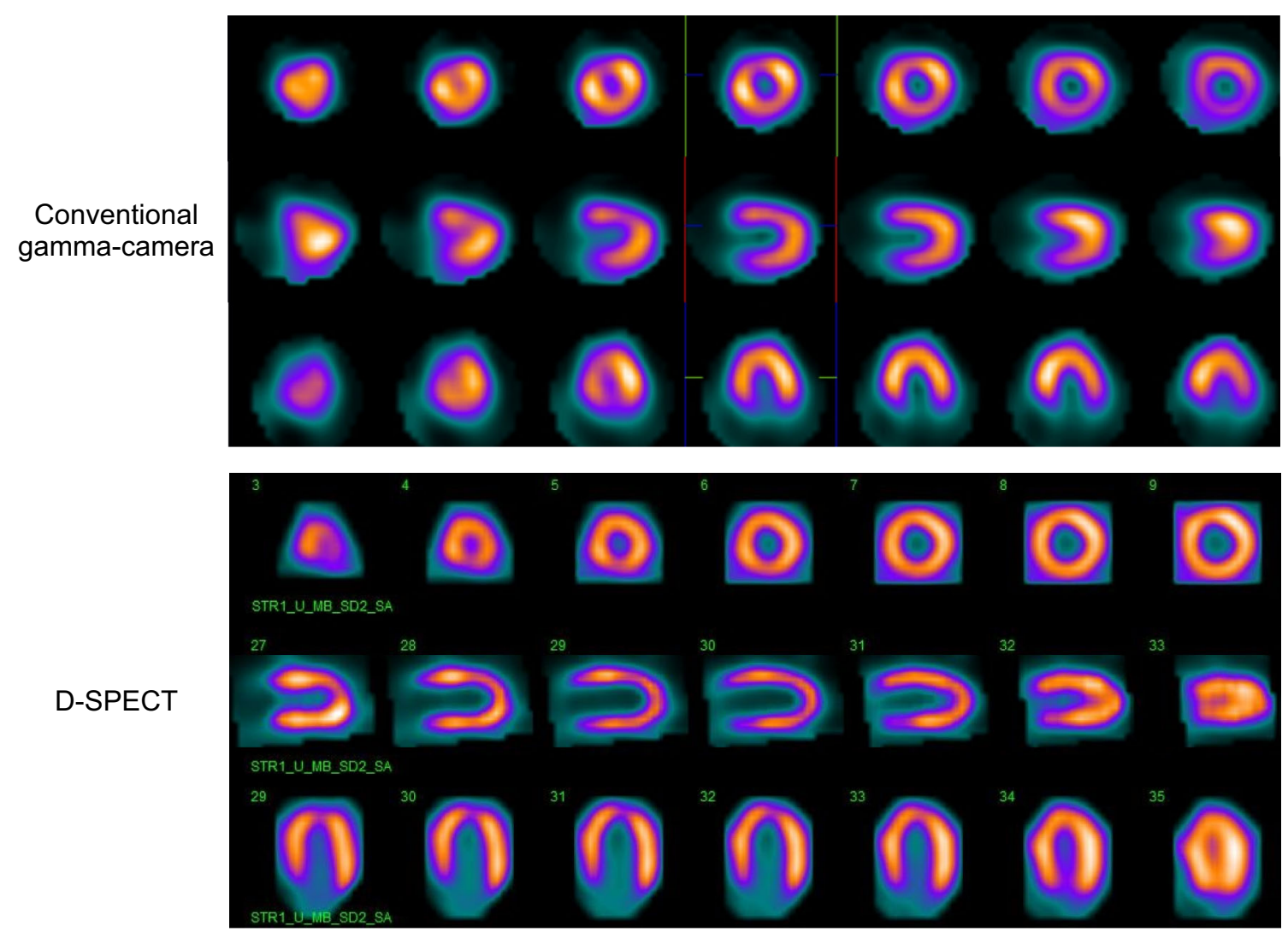

Fig. 3 Comparison between stress MPS images of a 48-year-old woman with hypertension, dyslipidemia and familiarity for CAD acquired with two different gamma cameras. The images acquired with a conventional dual-head GP gamma camera reveal a defect in anterior wall due to attenuation by left breast (top), while the stress images with a dedicated CZT gamma camera (D-SPECT) and upright patient position show homogenous left ventricular perfusion (bottom) 

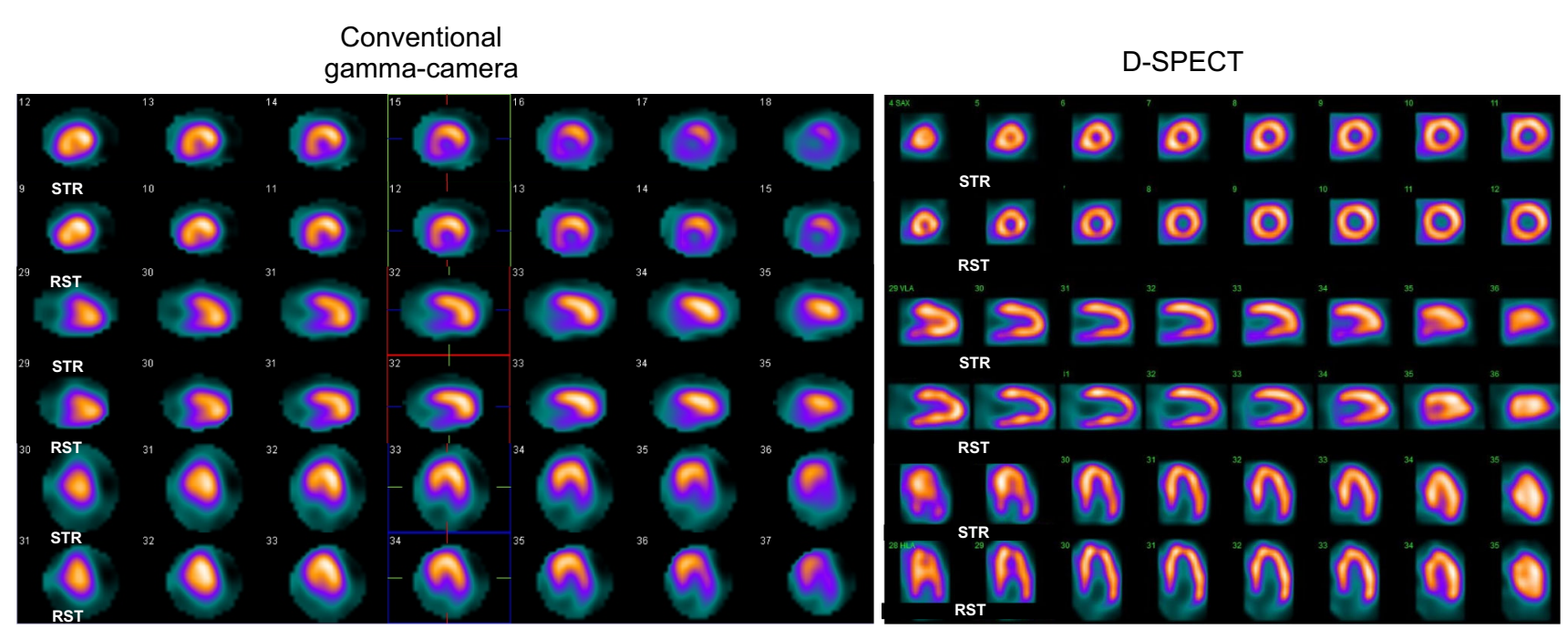

Fig. 4 Comparison between stress and rest MPS images of an obese 56 -year-old man (BMI $35.5 \mathrm{~kg} / \mathrm{m}^{2}$ ) with suspected CAD. The stress images acquired with a conventional dual-head GP gamma camera show a fixed inferior wall perfusion defect due to diaphragmatic

complete the full MPS examination, and that it had excellent prognostic value [36]. One-day stress-first MPS with $50 \%$ radiation reduction and a very low stress dose $(<2 \mathrm{mSv})$ using CZT technology and quantitative supine and prone analysis provided a high diagnostic value, similar to standard dose MPS [37].

However, few studies adapted the injected activities to patient weight or BMI. In the study by Hindorf et al., patients received an intravenous injection of $2.5 \mathrm{MBq} \mathrm{kg}{ }^{-1}$ body weight of ${ }^{99 \mathrm{~m}} \mathrm{Tc}$-tetrofosmin for the stress examination. The rest examination was performed only when the stress images were interpreted as abnormal with an injection of $4 \mathrm{MBq} \mathrm{kg}^{-1}$ [33]. Very recently, Oddstig et al. demonstrated that the linear low-dose weight-adjusted protocol of $2.5 \mathrm{MBq} / \mathrm{kg}$ can be applied over a wide range of body weights (51-193 kg, BMI 18-58) without loss of counts or image quality, and resulting in a significant reduction in radiation exposure to obese patients. In the case of a patient weighing $120 \mathrm{~kg}$, the effective dose for the stress examination would decrease to $2.1 \mathrm{mSv}$ (300 MBq) [38].

The CZT systems are available with up to 64-slice CT configuration (GE NM 570c). Very recently, Palyo et al. demonstrated that an ultra-low dose $(<190 \mathrm{MBq})$ in the stress examination, even with short imaging times ( $<6 \mathrm{~min})$, is feasible using a hybrid CZT-SPECT/CT camera without compromising image quality or significantly altering quantification of myocardial perfusion or left ventricular function [39].

However, many institutions may not have access to a CZT-SPECT system. Although these novel scanners offer high-quality imaging with a low radiation dose, they attenuation (left), while the stress/rest images with a dedicated CZT gamma camera (D-SPECT) and upright patient position show normal perfusion (right)

remain expensive, and are used in the clinical setting only for cardiac imaging [40].

\section{Conclusion}

Using innovative gamma cameras, collimators and software, as well as, unless otherwise indicated, stress-first imaging protocols, it has become possible to reduce significantly the effective dose in a high percentage of patients.

By combining new SPECT technologies with X-rayCT-based AC, MPS stress-only studies can conveniently be performed and likely reduce the incidence of attenuation artifacts. Although single patients may actually receive an increased effective dose due to $\mathrm{X}$-ray CT scanning, in the order of $1 \mathrm{mSv}$ when the CT scan is optimized for AC, application of the stress-first protocol (SPECT $+\mathrm{CT}$ ) allows the reduction of the dose over the entire population of patients undergoing MPS evaluation, since a conclusive normal stress study will exclude coronary artery disease and eliminate the need to perform a rest examination. On the other hand, an abnormal MPS will still require a rest evaluation to differentiate ischemia from scar. To further reduce the dose to the patients, the rest examination could be acquired without AC. In this case, the rest images should be compared with the corresponding non-corrected stress images.

Compliance with ethics guidelines The authors declare that they have no conflict of interest. This article does not contain any studies with human participants or animals performed by any of the authors. 
Open Access This article is distributed under the terms of the Creative Commons Attribution 4.0 International License (http://crea tivecommons.org/licenses/by/4.0/), which permits unrestricted use, distribution, and reproduction in any medium, provided you give appropriate credit to the original author(s) and the source, provide a link to the Creative Commons license, and indicate if changes were made.

\section{References}

1. Picano E, Vañó E, Rehani MM, Cuocolo A, Mont L, Bodi V et al (2014) The appropriate and justified use of medical radiation in cardiovascular imaging: a position document of the ESC Associations of Cardiovascular Imaging, Percutaneous Cardiovascular Interventions and Electrophysiology. Eur Heart J 35:665-672

2. Le Guludec D, Aigueperse J (2016) Dose optimization: a major challenge for acceptability of nuclear medicine. Clin Transl Imaging. 4:73-74

3. Fazel R, Krumholz HM, Wang Y, Ross JS, Chen J, Ting HH et al (2009) Exposure to low-dose ionizing radiation from medical imaging procedures. N Engl J Med 361:849-857

4. Cerqueira MD, Allman KC, Ficaro EP, Hansen CL, Nichols KJ, Thompson RC et al (2010) Recommendations for reducing radiation exposure in myocardial perfusion imaging. J Nucl Cardiol. 17:709-718

5. Slomka PJ, Dey D, Duvall WL, Henzlova MJ, Berman DS, Germano G (2012) Advances in nuclear cardiac instrumentation with a view towards reduced radiation exposure. Curr Cardiol Rep 14:208-216

6. Duvall WL, Rai M, Ahlberg AW, O'Sullivan DM, Henzlova MJ (2015) A multi-center assessment of the temporal trends in myocardial perfusion imaging. J Nucl Cardiol. 22:539-551

7. Henzlova MJ, Duvall WL, Einstein AJ, Travin MI, Verberne HJ (2016) ASNC imaging guidelines for SPECT nuclear cardiology procedures: Stress, protocols, and tracers. J Nucl Cardiol. 23:606-639

8. DePuey EG, Mahmarian JJ, Miller TD, Einstein AJ, Hansen CL, Holly TA et al (2012) Patient-centered imaging. J Nucl Cardiol. 19:185-215

9. Cuocolo A (2016) Challenges and opportunities of noninvasive cardiac imaging in obesity. J Nucl Cardiol. doi:10.1007/s12350016-0676-Z

10. Ferrari M, De Marco P, Origgi D, Pedroli G (2014) SPECT/CT radiation dosimetry. Clin Transl Imaging. 2:557-569

11. Einstein AJ, Johnson LL, Bokhari S, Son J, Thompson RC, Bateman TM et al (2010) Agreement of visual estimation of coronary artery calcium from low-dose CT attenuation correction scans in hybrid PET/CT and SPECT/CT with standard Agatston score. J Am Coll Cardiol 56:1914-1921

12. Cuocolo A (2011) Attenuation correction for myocardial perfusion SPECT imaging: still a controversial issue. Eur J Nucl Med Mol Imaging 38:1887-1889

13. Pellegrino T, Piscopo V, Boemio A, Russo B, De Matteis G, Pellegrino $S$ et al (2015) Impact of obesity and acquisition protocol on (123)I-metaiodobenzylguanidine indexes of cardiac sympathetic innervation. Quant Imaging Med Surg. 5:822-828

14. Hussain N, Parker MW, Henzlova MJ, Duvall WL (2016) Stressfirst Myocardial Perfusion Imaging. Cardiol Clin 34:59-67

15. Zoccarato O, Scabbio C, De Ponti E, Matheoud R, Leva L, Morzenti S et al (2014) Comparative analysis of iterative reconstruction algorithms with resolution recovery for cardiac SPECT studies. A multi-center phantom study. J Nucl Cardiol. 21:135-148
16. Zoccarato O, Marcassa C, Lizio D, Leva L, Lucignani G, Savi A et al (2016) Differences in polar-map patterns using the novel technologies for myocardial perfusion imaging. J Nucl Cardiol. doi:10.1007/s12350-016-0500-9

17. Lecchi M, Martinelli I, Zoccarato O, Maioli C, Lucignani G, Del Sole A (2016) Comparative analysis of full-time, half-time, and quarter-time myocardial ECG-gated SPECT quantification in normal-weight and overweight patients. J Nucl Cardiol. doi:10. 1007/s12350-015-0382-2

18. Valenta I, Treyer V, Husmann L, Gaemperli O, Schindler MJ, Herzog BA et al (2010) New reconstruction algorithm allows shortened acquisition time for myocardial perfusion SPECT. Eur J Nucl Med Mol Imaging 37:750-757

19. DePuey EG, Bommireddipalli S, Clark J, Leykekhman A, Thompson LB, Friedman M (2011) A comparison of the image quality of full-time myocardial perfusion SPECT vs wide beam reconstruction half-time and half-dose SPECT. J Nucl Cardiol. 18:273-280

20. Marcassa C, Campini R, Zoccarato O, Calza P (2011) Wide beam reconstruction for half-dose or half-time cardiac gated SPECT acquisitions: optimization of resources and reduction in radiation exposure. Eur J Nucl Med Mol Imaging 38:499-508

21. Bateman TM, Heller GV, McGhie AI, Courter SA, Golub RA, Case JA et al (2009) Multicenter investigation comparing a highly efficient half-time stress-only attenuation correction approach against standard rest-stress Tc-99 m SPECT imaging. J Nucl Cardiol. 16:726-735

22. Marcassa C, Zoccarato O, Calza P, Campini R (2013) Temporal evolution of administered activity in cardiac gated SPECT and patients' effective dose: analysis of an historical series. Eur J Nucl Med Mol Imaging 40:325-330

23. Caobelli F, Kaiser SR, Thackeray JT, Bengel FM, Chieregato M, Soffientini A et al (2014) IQ SPECT allows a significant reduction in administered dose and acquisition time for myocardial perfusion imaging: evidence from a phantom study. J Nucl Med 55:2064-2070

24. Gremillet E, Agostini D (2016) How to use cardiac IQ•SPECT routinely? An overview of tips and tricks from practical experience to the literature. Eur J Nucl Med Mol Imaging 43:707-710

25. Lyon MC, Foster C, Ding X, Dorbala S, Spence D, Bhattacharya $M$ et al (2016) Dose reduction in half-time myocardial perfusion SPECT-CT with multifocal collimation. J Nucl Cardiol. 23:657-667

26. Imbert L, Perrin M, Verger A, Marie P-Y (2016) Dose optimization for myocardial perfusion CZT-SPECT imaging: why and how? Clin Transl Imaging. 4:13-20

27. Erlandsson K, Kacperski K, van Gramberg D, Hutton BF (2009) Performance evaluation of D-SPECT: a novel SPECT system for nuclear cardiology. Phys Med Biol 54:2635-2649

28. Bocher M, Blevis IM, Tsukerman L, Shrem Y, Kovalski G, Volokh L (2010) A fast cardiac gamma camera with dynamic SPECT capabilities: design, system validation and future potential. Eur J Nucl Med Mol Imaging 37:1887-1902

29. Zoccarato O, Lizio D, Savi A, Indovina L, Scabbio C, Leva L et al (2016) Comparative analysis of cadmium-zincum-telluride cameras dedicated to myocardial perfusion SPECT: a phantom study. J Nucl Cardiol. 23:885-893

30. Duvall WL, Croft LB, Godiwala T, Ginsberg E, George T, Henzlova MJ (2010) Reduced isotope dose with rapid SPECT MPI imaging: initial experience with a CZT SPECT camera. J Nucl Cardiol 17:1009-1014

31. Gunalp B (2015) Role of cardiac ultrafast cameras with CZT solid-state detectors and software developments on radiation absorbed dose reduction to the patients. Radiat Prot Dosimetry $165: 461-463$ 
32. Gimelli A, Bottai M, Quaranta A, Giorgetti A, Genovesi D, Marzullo P (2013) Gender differences in the evaluation of coronary artery disease with a cadmium-zinc telluride camera. Eur J Nucl Med Mol Imaging 40:1542-1548

33. Hindorf C, Oddstig J, Hedeer F, Hansson MJ, Jögi J, Engblom H (2014) Importance of correct patient positioning in myocardial perfusion SPECT when using a CZT camera. J Nucl Cardiol 21:695-702

34. Perrin M, Djaballah W, Moulin F, Claudin M, Veran N, Imbert L, et al (2015) Stress-first protocol for myocardial perfusion SPECT imaging with semiconductor cameras: high diagnostic performances with significant reduction in patient radiation doses. Eur $\mathbf{J}$ Nucl Med Mol Imaging 1004-1011

35. Chaudhry W, Hussain N, Ahlberg AW, Croft LB, Fernandez AB, Parker MW et al (2015) Multicenter evaluation of stress-first myocardial perfusion image triage by nuclear technologists and automated quantification. J Nucl Cardiol. doi:10.1007/s12350015-0291-4

36. Einstein AJ, Johnson LL, DeLuca AJ, Kontak AC, Groves DW, Stant J et al (2015) Radiation dose and prognosis of ultra-low- dose stress-first myocardial perfusion SPECT in patients with chest pain using a high-efficiency camera. J Nucl Med 56:545-551

37. Sharir T, Pinskiy M, Pardes A, Rochman A, Prokhorov V, Kovalski G et al (2016) Comparison of the diagnostic accuracies of very low stress-dose with standard-dose myocardial perfusion imaging: automated quantification of one-day, stress-first SPECT using a CZT camera. J Nucl Cardiol 23:11-20

38. Oddstig J, Hindorf C, Hedeer F, Jögi J, Arheden H, Hansson MJ et al (2016) The radiation dose to overweighted patients undergoing myocardial perfusion SPECT can be significantly reduced: validation of a linear weight-adjusted activity administration protocol. J Nucl Cardiol. doi:10.1007/s12350-016-0628-7

39. Palyo RJ, Sinusas AJ, Liu Y-H (2016) High-sensitivity and highresolution SPECT/CT systems provide substantial dose reduction without compromising quantitative precision for assessment of myocardial perfusion and function. J Nucl Med 57:893-899

40. DePuey EG (2016) Traditional gamma cameras are preferred. J Nucl Cardiol. 23:795-802 